\title{
VISÃO DO ENFERMEIRO FRENTE À PRÁTICA DA EDUCAÇÃO EM SAÚDE NO AMBIENTE HOSPITALAR*
}

Aline Belletti Figueira' ${ }^{1}$ Simone Coelho Amestoy², Diana Cecagno³ , Fernanda Sant’Ana Tristão, Letícia de Lima Trindade ${ }^{5}$, Veridiana Ávila Correa ${ }^{1}$

RESUMO: Este estudo teve como objetivo conhecer a visão do enfermeiro frente à prática da educação em saúde no ambiente hospitalar. Trata-se de uma pesquisa com abordagem qualitativa, do tipo descritiva e exploratória, na qual participaram sete enfermeiras que trabalham em unidades de internação de um hospital de médio porte localizado na Região Sul do Rio Grande do Sul. Os dados foram coletados por meio de entrevistas semiestruturadas e observação não participante e analisados com auxílio da Análise de Conteúdo Temática, emergindo três categorias: Educação em saúde na prática das enfermeiras; Dificuldades para a realização da educação em saúdeno ambiente hospitalar e Estratégias para a implementação da educação em saúde. As participantes acreditam na relevância da educação em saúde e buscam inferi-la em seu cotidiano. A falta de investimento na qualificação dos profissionais e a sobrecarga de atividades foram identificadas como os principais problemas institucionais que dificultam seu exercício. DESCRITORES: Enfermagem; Educação em saúde; Formação de recursos humanos.

\section{THE VISION OF THE NURSES IN THE FACE OF THE PRACTICE OF HEALTH EDUCATION IN THE HOSPITAL ENVIRONMENT}

ABSTRACT: This study aimed to investigate the vision of the nurse in the face of the practice of health education in the hospital environment. This is research with a qualitative approach, of the descriptive and exploratory type, in which seven nurses participated. These work on the wards of a medium-sized hospital located in the Southern Region of Rio Grande do Sul. The data was collected through semi-structured interviews and non-participant observation, and was analyzed with the help of Thematic Content Analysis. Three categories emerged: Health education in the nurses' practice; Difficulties in carrying out health education in the hospital environment and Strategies for implementing health education. The participants believe in the relevance of health education and seek to insert it in their routine. The lack of investment in training the professionals and the overload of activities were identified as the principal institutional problems which impede the exercising of health education. DESCRIPTORS: Nursing; Health education; Training of human resources.

\section{VISIÓN DEL ENFERMERO DELANTE DE LA PRÁCTICA DE EDUCACIÓN EN SALUD EN EL AMBIENTE HOSPITALAR}

RESUMEN: Este estudio tuvo como objetivo conocer la visión del enfermero delante de la práctica de educación en salud en el ambiente hospitalar. Es una investigación con abordaje cualitativo, del tipo descriptivo y exploratorio, en la cual participaron siete enfermeras que trabajan en unidades de internación de un hospital de medio porte ubicado en la Región Sur de Rio Grande do Sul. Los datos fueron obtenidos por medio de entrevistas semiestructuradas y observación no participante y analizados con ayuda del Análisis de Contenido Temática, resultando en tres categorías: Educación en salud en la práctica de los enfermeros; Dificultades para la realización de la educación en salud en el ambiente hospitalar y Estrategias para la implementación de la educación en salud. Las participantes creen en la relevancia de la educación en salud y buscan inferirla en su cotidiano. La falta de inversiones en la cualificación de los profesionales y la sobrecarga de actividades fueron identificadas como los principais problemas institucionales que dificultam su ejercicio.

DESCRIPTORES: Enfermería; Educación en salud; Formación de recursos humanos.

*Artigo desenvolvido a partir de monografia de graduação 'Visão do enfermeiro frente à utilização da educação em saúde no ambiente hospitalar' apresentada a Universidade Federal de Pelotas - UFPel, em 2012.

${ }^{1}$ Enfermeira.

${ }^{2}$ Enfermeira. Doutora em Enfermagem. Professora da Faculdade de Enfermagem da UFPel. Membro do Grupo de Pesquisa em Educação em Enfermagem e Saúde - EDEN e do Núcleo de Estudos e Pesquisas em Enfermagem - NEPEN.

${ }^{3}$ Enfermeira. Mestre em Enfermagem. Doutoranda pelo Programa de Pós-Graduação em Enfermagem Universidade Federal do Rio Grande. Professora da Faculdade de Enfermagem da UFPel.

${ }^{4}$ Enfermeira.MestreemEducação.DoutorandapeloProgramadePós-GraduaçãoemEnfermagemdaUFPel.ProfessoradaFaculdadedeEnfermagemdaUFPel. ${ }^{5}$ Enfermeira. Doutora em Enfermagem. Professora da Universidade do Estado de Santa Catarina - UDESC. Membro do Grupo Práxis da Universidade Federal de Santa Catarina. Líder do Grupo de Pesquisa sobre Saúde e Trabalho da UDESC.

Autor correspondente:

Recebido: 05/09/2012

Simone Coelho Amestoy

Aprovado: 21/02/2013

Universidade Federal de Pelotas

Rua Gomes Carneiro, 1 - 96010-610 - Pelotas-RS-Brasil

E-mail: simoneamestoy@hotmail.com

Cogitare Enferm. 2013 Abr/Jun; 18(2):310-6 


\section{INTRODUÇÃO}

Dentre as diversas atividades do enfermeiro na sociedade moderna, a educação em saúde desponta como uma das principais estratégias para fomento da promoção da saúde. Logo, a inserção da educação em saúde, no cotidiano de trabalho do enfermeiro, auxilia de modo fundamental na autonomia do ser cuidado ${ }^{(1)}$.

$\mathrm{O}$ avanço e mudança da nomenclatura, de educação para a saúde, para educação em saúde, revelam expectativas de ruptura dos paradigmas vigentes na prática educativa voltada a uma nova perspectiva de entender a saúde, comprometida com os princípios do Sistema Único de Saúde (SUS). A educação para a saúde baseava-se na concepção de que o indivíduo tinha que aprender a cuidar de sua saúde, vista como ausência de doença. Já a educação em saúde, fundamenta-se na concepção de que o indivíduo aprende a cuidar de sua saúde, que é resultante de múltiplos fatores inerentes ao processo saúde-doença, a partir do referencial coletivo de conhecimento de sua realidade ${ }^{(2)}$, amparada na busca de autonomia e qualidade de vida. No entanto, destaca-se que a mudança na nomenclatura não, necessariamente, expressa a ruptura com o modelo individualista que responsabiliza o indivíduo pela sua saúde. Essa depende da mudança de comportamento e de compreensão sobre o assunto e incorporação de novas práticas por parte dos profissionais de saúde e sociedade.

A evolução do conceito de educação em saúde acompanha a perspectiva difundida pelas concepções de promoção à saúde que podem ser reunidas em dois grandes grupos. O comportamental, de mudanças de estilo de vida e aquela que busca articular educação, condições e qualidade de vida ${ }^{(3)}$. Dessa forma, o conceito de educação em saúde está ancorado nas políticas de saúde, as quais tentam direcionar para o rompimento da hegemonia do modelo biomédico, vislumbra a intensificação de ações de promoção no cotidiano dos serviços de saúde, o desenvolvimento da autonomia dos indivíduos, profissionais e coletividades. Isso para que em conjunto possam compreender a saúde como resultante das condições de vida e propiciar um desenvolvimento social mais equitativo(4).

Complementa-se que a educação em saúde constitui-se em um conjunto de práticas pedagógicas e sociais, de conteúdo técnico, político e científico. No âmbito das ações à saúde, a educação deve ser vivenciada e compartilhada pelos trabalhadores da área, pelos setores organizados da população e usuários. A educação em saúde representa um processo sistemático e permanente que objetiva a formação e o desenvolvimento da consciência crítica do cidadão $^{(5)}$. Nesse sentido, torna-se primordial que o enfermeiro realize educação em saúde, de forma comprometida com os princípios de cidadania e democracia, auxiliando na emancipação do indivíduo como sujeito histórico e social capaz de propor e opinar nas decisões de saúde, evidenciando o compromisso social com a melhoria do estado de saúde da população $0^{(6)}$. Nesse contexto, a presente pesquisa objetivou conhecer a visão do enfermeiro frente à prática da educação em saúde no ambiente hospitalar.

\section{MÉTODO}

Trata-se de uma pesquisa com abordagem qualitativa, do tipo descritiva e exploratória e foi realizado nos meses de março a maio de 2012. O estudo contou com a participação de sete enfermeiras que trabalham em unidades de internação de um hospital de médio porte localizado na Região Sul do Rio Grande do Sul, que tiveram interesse em discutir e refletir sobre o tema educação em saúde no ambiente hospitalar. Destaca-se que o hospital possui 10 enfermeiras nas unidades de internação, sendo que destas, seis trabalham oito horas diárias. Ao convidá-las para participar do estudo, três recusaram alegando falta de tempo, em virtude da sobrecarga de trabalho.

Para a coleta das informações foram utilizadas entrevistas semiestruturadas e observação não participante. As entrevistas foram realizadas pelas pesquisadoras no próprio local do estudo, em encontros individuais, a partir de um roteiro semiestruturado. As entrevistas foram nomeadas de acordo com a ordem de sua realização, sendo: E1, E2...E7.

Ainda, realizaram-se observações não participantes focalizadas para a melhor compreensão da forma com que as enfermeiras realizam ações de educação em saúde na assistência cotidiana, bem como atentar para as estratégias utilizadas. Realizaram-se, aproximadamente, três horas de observação das atividades de cada enfermeira, totalizando 21 horas de observação. Os achados emergidos da observação foram identificados pela letra "O”, seguida do número ordinal correspondente a sua realização $(\mathrm{O} 1, \mathrm{O} 2 \ldots \mathrm{O} 7)$.

As informações coletadas foram analisadas a partir do agrupamento das ideias, elementos e manifestações ao redor de uma temática capaz de estabelecer uma classificação, para tanto, utilizou-se a Análise de Conteúdo Temática ${ }^{(7)}$. 
Convém informar que foram respeitadas as recomendações da Resolução 196/96 do Conselho Nacional de Saúde, que regulamenta investigações envolvendo seres humanos. A pesquisa foi submetida e aprovada pelo Comitê de Ética em Pesquisa, aprovada com o protocolo número 176/2012.

\section{RESULTADOS}

Participaram do estudo sete enfermeiras com idade entre 35 e 27 anos, com tempo de formação entre três e doze anos, e tempo de trabalho na instituição entre três e dez anos. Quanto à formação uma faz mestrado, três possuem especialização e três delas possuem graduação exclusiva. A partir da análise das informações emergiram três categorias, apresentadas a seguir.

\section{Educação em saúde na prática das enfermeiras}

Essa categoria representa a visão das enfermeiras frente à prática da educação em saúde no ambiente hospitalar. As participantes relataram, como exemplificado a seguir, a relevância dessa atividade no desenvolvimento de um trabalho efetivo e de qualidade para todos os envolvidos, a qual deve ser valorizada durante a formação acadêmica.

[...] a formação do enfermeiro abrange a educação, o processo de educação, a formação técnica não abrange tanto. Então, é o enfermeiro que precisa de um tempo maior pra treinar bem sua equipe para esse processo, para que então depois eles sejam multiplicadores do teu trabalho. (E6)

Parte das enfermeiras enfatizou também a relevância da educação em saúde, nos diversos serviços da rede assistencial, ressaltando a importância de não restringir a mesma à Atenção Básica em Saúde:

Eu acho que é bastante relevante, eu acho que é muito importante, mesmo dentro de um hospital, a educação do paciente. Acho que existe ainda essa mentalidade de à educação ser feita mais em pré-hospitalar, lá na unidade básica, na saúde da família, mas essa educação em saúde se faz com certeza no hospital também. (E3)

É um processo realizado desde o momento que o paciente interna até sua alta hospitalar. (E5)
O recorte a seguir demonstra que a valorização do corpo biológico ainda permeia as condutas profissionais, em detrimento à compreensão do ser humano enquanto ser biopsicossocioespiritual:

A enfermeira entra no quarto de um senhor internado, pede para visualizar a úlcera da pressão localizada no cóccix. Após, orienta o paciente sobre a importância de seguir uma boa alimentação, o que irá contribuir para a recuperação mais rápida. $(\mathrm{O} 2)$

Cabe reforçar que as enfermeiras que participaram da pesquisa compreendem como importante a prática da educação em saúde no ambiente hospitalar. Porém, observa-se a replicação de um modelo de educação voltado para práticas higienistas, em que o conhecimento é transmitido de forma vertical, caracterizado pela ausência de mecanismos democráticos, ignorando a integração e articulação de diferentes saberes e práticas.

\section{Dificuldades para a realização da educação em saúde no ambiente hospitalar}

Ao longo do estudo verificou-se que algumas enfermeiras encontram diversas barreiras ao trabalhar com o tema no seu cotidiano. As participantes atribuem à dificuldade de realizar a educação em saúde à falta de pessoal e de tempo, devido a se sentirem sobrecarregadas de atividades, como apresentado a seguir:

No hospital os funcionários são muito exigidos, por exemplo, se tu pegar uma unidade que tem muitos pacientes e poucos funcionários, tipo pós-cirúrgicos, ou pacientes com cuidados clínicos. Não tem aqui funcionário suficiente prá trabalhar. Está sempre faltando, o hospital quer que a gente faça um bom trabalho, mas não tem pessoal, sobrecarrega entende! (E1)

Acho que é cultural, assim, acho que tem profissionais que querem fazer educação em saúde, mas o problema é a rotina, a sobrecarga de atividades. (E4)

Além disso, na visão das enfermeiras a educação em saúde é pouco incentivada e valorizada na instituição em que atuam:

Na instituição o tema educação em saúde não é trabalhado. Existe um grupo de educação, mas voltado aos profissionais, não existe nenhum trabalho, pelo menos eu não sou sabedora de nenhum trabalho voltado prá 
educação em saúde com os pacientes, mesmo em unidades de internação cirúrgica, são unidades em que o atendimento de educação com o paciente. Eu acho que até exista, mas ele não está formalizada. (E6)

A partir dos relatos evidenciou-se certa dificuldade das participantes de inserir práticas de educação em saúde em seu local de trabalho. Acredita-se que este fato está relacionado à forma de compreender o hospital. Este como um espaço inerte à educação em saúde, vinculado a sua constituição e organização histórica com relações de poder rígidas, voltada para o tecnicismo e medicalização da saúde, que foram sendo naturalizados no espaço hospitalar pelo discurso biomédico.

\section{Estratégias para a implementação da educação em saúde no ambiente hospitalar}

Emergiu no estudo duas estratégias principais para a realização da educação em saúde, sendo elas: a formação do vínculo com a família e o diálogo.

Destacou-se a importância de se estabelecer bom envolvimento com a família dos usuários, o qual facilitaria a comunicação e a troca de saberes, bem como qualificaria a assistência realizada, uma vez que traz satisfação ao usuário/familiar, como o recorte exemplifica:

A família precisa estar inserida nesse contexto de aprendizado, para poder ajudar, se sentir recompensada em estar participando disso, se sentindo valorizada. (E6)

No que concerne o diálogo destaca-se o seguinte depoimento:

Eu faço visitação diária prá todos os pacientes. Então, todos os dias eu visito paciente por paciente e faço uma conversação, diálogo com cada um. Pergunto como é que eles estão, eu converso sobre seu problema, busco sanar dúvidas, conforme eu posso. (E3)

Evidenciou-se a comunicação como ferramenta primordial para promoção da educação em saúde, uma vez que essa depende da reciprocidade entre profissionais e usuários, bem como da formação de vínculo entre os mesmos.

\section{DISCUSSÃO}

O enfermeiro pode ser compreendido como um educador em saúde, e a educação em saúde uma ferramenta inerente à profissão. No entanto, a forma como esta prática vem sendo inserida no ambiente hospitalar gera a necessidade de revê-la e discutí-la, constantemente.

No contexto atual, critica-se a concepção positivista, que compreende a educação em saúde como uma prática reducionista, fundamentada em ensinamentos impostos, prescritivos. Isso com a finalidade de estimular a adesão dos sujeitos sociais a comportamentos, desvinculados de sua realidade, tornando-os simples objetos passivos das intervenções, que em geral são preconceituosas, coercitivas e punitivas. Todavia, o esperado é uma educação que fomente a participação ativa da comunidade, proporcionando informação, educação sanitária e promovendo as atitudes indispensáveis para a vida ${ }^{(8)}$.

Ao analisar o trabalho exercido no ambiente hospitalar, os participantes do estudo destacam a importância de inserir, de forma consistente, a educação em saúde na instituição, valorizando sua relevância em toda a rede de saúde. Os achados também apontam para a importância de desenvolver atividades de educação em saúde durante a formação do enfermeiro, observando e valorizando o papel primordial das mesmas nos diversos cenários de ensino-aprendizagem, bem como nas ações de extensão e pesquisa. Para tanto, a mesma deve favorecer as práticas educacionais que potencializem o empoderamento dos sujeitos para atuarem na efetivação das mudanças sociais ${ }^{(9)}$. Sobre isso, autores lembram que por mais que se deseja inovar no campo da promoção da saúde, torna-se difícil contribuir com a implementação de novas propostas, se não houver investimentos em mudanças na formação $0^{(10)}$.

O estudo revela que os enfermeiros em seus discursos valorizam as atividades de educação em saúde. Contudo, as observações permitem afirmar que os mesmos têm dificuldade de efetivar essa prática, atuando mediante orientações pontuais, prescritivas e focadas na doença, sinalizando o predomínio do antigo modelo voltado para a promoção da saúde e pela influência do modelo flexneriano. Este modelo enfatiza o enfoque biologicista de doença, negando-se a determinação social da saúde; com grande destaque à disciplinaridade e abordagem reducionista do conhecimento, no qual o corpo humano é compreendido como uma máquina. O modelo é considerado, do ponto de vista pedagógico, como um modelo massificador, hospitalocêntrico, individualista, atribuindo ao indivíduo a responsabilidade por sua doença e com forte tendência à especialização, gerando efeitos prejudiciais a formação profissional em saúde ${ }^{(11)}$. 
Frente aos achados, identificou-se que as enfermeiras dentre a diversidade de atividades que desenvolvem, assumem pouca responsabilidade diante da educação em saúde. Mesmo realizando-a em seu cotidiano de trabalho hospitalar, elas não reconhecem como uma atividade que pode ser desenvolvida neste espaço, tem-se a ideia de que a prática está incorporada somente na atenção básica, aspecto que precisa ser mais discutido e refletido entre as participantes.

A educação em saúde, desenvolvida em nível de internação hospitalar, representa uma tendência emergente na enfermagem. No entanto necessita ser investigada, criada e recriada, promovendo interfaces entre o ensino, a pesquisa, a assistência e a gerência, por meio de um movimento de construção do conhecimento e de desenvolvimento da Ciência da Enfermagem ${ }^{(12)}$.

Identifica-se o hospital como um espaço terapêutico e de formação e produção de saberes científicos, no qual se estabelece uma relação direta entre o saber cuidar do corpo e o poder de cuidá-lo, resultando na noção de propriedade do corpo instituída nos hospitais na modernidade. Apesar do corpo se impor de forma indiscutível no hospital, por dar visibilidade a uma rede de saberes, o mesmo é compreendido como algo que pode ser medido, constatado e combatido, que funciona ou não funciona, como se fosse uma máqui$\mathrm{na}^{(13)}$. Esta forma de compreensão do corpo institucionalizado, ainda presente no cenário atual, alerta para a necessidade de repensar as práticas de saúde, tanto assistenciais como educativas, por distanciarem-se das políticas públicas de saúde, as quais buscam minimizar iniquidades e promover saúde enquanto direito.

A educação em saúde vem a ser um instrumento capaz de transformar a prática profissional, conferindo ao cuidado características de uma atividade crítica e criativa, mostrando a necessidade de ser bem desenvolvida pelos profissionais da saúde ${ }^{(14)}$.

Um estudo relembra que, frequentemente, a educação em saúde tem se restringido a práticas higienistas, palestras prescritivas de hábitos e condutas, tratando a população usuária de forma passiva, transmitindo conhecimentos técnicos sobre as doenças e como cuidar da saúde, sem levar em conta o saber popular e as condições de vida dos indivíduos assistidos ${ }^{(12)}$.

Os espaços restritos para o diálogo favorecem a desvalorização do conhecimento dos usuários, e as ações educativas passam a replicar o modelo da educação bancária, a qual representa a simples transmissão de conhecimento, com escassa abertura para um processo comunicacional permeado pelo dialógico ${ }^{(15)}$.
É este modelo tradicional de educação, prescritivo e anulador do pensamento crítico, que precisa ser superado na prática. Sabe-se que não se trata de uma tarefa fácil, mas sim um desafio, no qual o enfermeiro necessita ao realizar atividades de educação em saúde no ambiente hospitalar, envolver os usuários, buscando estimular a autonomia e o autocuidado.

O estudo não tem a pretensão de dar receitas prontas, mesmo porque, isso vai contra a educação aqui defendida, ou seja, uma educação problematizadora, construída a partir da valorização das pessoas, enquanto sujeitos sociais e históricos, levando-se em consideração sua realidade e cultura. Assim, o educador não somente educa, mas enquanto educa é educado, por meio do estabelecimento do diálogo com o educando que, por sua vez, ao ser educado também educa ${ }^{(15)}$. Aqui reside uma grande diferença, entre o enfermeiro que se restringe a dar orientações e o enfermeiro que busca estimular a cidadania e o senso crítico dos indivíduos, a fim de que as mesmas possam optar por decisões saudáveis capazes de contribuir com sua qualidade de vida.

Entre as dificuldades encontradas, identificadas pelos próprios participantes, emergiram aspectos relacionados às condições de trabalho que geram sobrecarga de trabalho, falta de valorização das práticas de educação em saúde e de incentivos por parte dos gestores. Em relação à falta de recursos humanos, sobrecarga de trabalho, pouco incentivo da instituição para a realização de atividades educativas e dificuldades de compreensão dos usuários, entre os aspectos que dificultam a inserção da educação em saúde no ambiente hospitalar, entende-se que esses tem repercussão na assistência prestada. Por vezes, também são utilizados como justificativas para a fragilidade ou ausência de ações de educação em saúde. A partir deste resultado, também se evidencia a necessidade de promover um espaço de discussão envolvendo os gestores, pois a inserção efetiva da educação em saúde no ambiente hospitalar depende das condições de trabalho oferecidas e da forma como a instituição vislumbra essa atividade.

Nas últimas décadas podem ser identificadas mudanças nas ações do enfermeiro em função das necessidades dos serviços de saúde, levando-o a assumir uma multiplicidade de atividades, as quais geram o afastamento gradativo desse profissional em relação ao cuidado, contribuindo para o afastamento do enfermeiro de seu papel de educador e agente de transformação ${ }^{(16)}$.

Além das contribuições postas pelos sujeitos para fortalecimento da educação em saúde, resgata-se a pers- 
pectiva da integralidade, a qual inclui políticas públicas, ambientes apropriados para além dos tratamentos clínicos e curativos, comprometidas com o desenvolvimento da solidariedade e da cidadania, envolvidas na melhoria da qualidade de vida ${ }^{(3)}$ e na promoção da saúde dos indivíduos. No entanto, cabe destacar que não adianta modificar o conceito de educação em saúde se as práticas educativas ainda estão debruçadas sobre velhos conceitos.

Sendo a educação em saúde visualizada como processo político pedagógico, essa retoma a necessidade de desenvolver um pensar crítico e reflexivo, permitindo desvelar a realidade, propondo ações transformadoras que levem o indivíduo a sua autonomia e emancipação enquanto sujeito histórico e social capaz de propor e opinar nas decisões de saúde para o cuidar de si, de sua família e da coletividade ${ }^{(3)}$.

Desta forma, ações educativas em saúde tornam-se um processo com objetivo de capacitar indivíduos, familiares ou grupos, com o intuito de contribuir na melhoria das condições de vida, bem como estimular a reflexão crítica sobre suas necessidades de saúde e ações requeridas para sua resolução ${ }^{(17)}$. Por esse motivo, a criação de vínculo também se torna necessária, com vistas a aproximar os diferentes sujeitos envolvidos no cuidado, inclusive a família, frequentemente esquecida na assistência hospitalar, mas sinalizada pelos sujeitos como importante estratégia potencializado da educação em saúde nesse cenário.

Enfatiza-se a necessidade de incluir a família no cuidado, tornando-a elemento chave frente às práticas de educação em saúde. O apoio da equipe e a prática do diálogo entre os sujeitos envolvidos na hospitalização são importante para amenizar as fontes de desgastes dos usuários, norteando uma conduta mais humanizada e comprometida com suas necessidades ${ }^{(18)}$.

Vislumbra-se o diálogo, como estratégia que facilita a prática da educação em saúde no ambiente hospitalar. A partir desta perspectiva dialógica pode-se pensar em novas possibilidades de inserir a educação em saúde no ambiente hospitalar, compatíveis com o modelo de saúde vigente no país, o qual tem como prioridade a promoção da saúde.

\section{CONSIDERAÇÕES FINAIS}

Por meio da realização deste estudo, tornou-se possível conhecer a visão do enfermeiro frente à utilização da educação em saúde no ambiente hospitalar e identificou-se a importância desta atividade no cotidiano das participantes. Contudo, há dificuldades em consolidá-la na prática.
Acredita-se que as dificuldades em se trabalhar com educação em saúde no ambiente hospitalar estão relacionadas a pouca valorização que as próprias enfermeiras atribuem a tal atividade, como se a mesma fosse responsabilidade somente dos profissionais da atenção básica. Frente aos achados, destaca-se que tanto nos depoimentos como nas observações foi possível evidenciar a sobrecarga de trabalho como um fator que interfere de forma negativa na realização da educação em saúde. Mediante o exposto, urge a necessidade de expandir a discussão entre as demais enfermeiras do hospital e os gestores da instituição, a fim de sensibilizá-los quanto a sua relevância, disponibilizando condições de trabalho adequadas para sua efetiva inserção. Além disso, defende-se o investimento na educação dos novos profissionais. Educação que deve buscar provocar algumas rupturas no modelo biomédico, ainda hegemônico e dominante em muitas instituições de saúde.

Também foi possível identificar que o tema carece de mais investimentos em pesquisa, a fim de sensibilizar os gestores e profissionais da saúde quanto à magnitude do mesmo, bem como avanços que pode promover a qualidade da assistência hospitalar.

\section{REFERÊNCIAS}

1. Souza LM, Wegner W, Gorini MIPO. Educação em saúde: uma estratégia de cuidado ao cuidador leigo. Rev. Latino-Am. Enfermagem. [Internet] 2007;15(2) [acesso em 20 mai 2012]. Disponível: http://dx.doi.org/10.1590/ S0104-11692007000200022

2. Pelicioni MCF, Pelicioni AF. Educação e promoção da saúde: uma retrospectiva histórica. Mundo Saúde. [Internet] 2007;31(3) [acesso em 10 abr 2012]. Disponível: http://www.scamilo.edu.br/pdf/mundo_ saude/55/02_restrospectiva_historica.pdf

3. Buss PM. Uma introdução ao conceito de promoção da saúde. In: Czeresina D, Freitas CM, organizadores. Promoção da Saúde. Rio de Janeiro: Fiocruz; 2003. P. 15-38.

4. Heidmann ITS, Buss A, Puntel MC, Boehs AE, Wosny AM, Monticelli M. Promoção à saúde: trajetória histórica de suas concepções. Texto Contexto Enferm. [Internet] 2006;15(2) [acesso em 05 abr 2012]. Disponível: http:// dx.doi.org/10.1590/S0104-072006000200021

5. Brasil. Fundação Nacional de Saúde. Diretrizes de educação em saúde visando à promoção da saúde: 
documento base - documento I / Fundação Nacional de Saúde - Brasília: Funasa; 2008. Disponível: http://www. funasa.gov.br/site/wp-content/files_mf/dir_ed_sau.pdf

6. Monteiro EMLM, Vieira NFC. Educação em saúde a partir de círculos de cultura. Rev Bras Enferm. [Internet] 2010;63(3) [acesso em 10 abr 2012]. Disponível: http:// dx.doi.org/10.1590/S0034-71672010000300008

7. Minayo MCS. O desafio do conhecimento: pesquisa qualitativa em saúde. 10a ed. São Paulo: Hucitec; 2007.

8. Pedrosa JIS. Educação popular no Ministério da Saúde: identificando espaços e referências. In: Brasil, Ministério da Saúde. Caderno de educação popular em saúde. Brasília: Ministério da Saúde; 2007.

9. Silva KL, Senna RR, Grillo MJC, Horta NC, Prado PMC. Educação em enfermagem e os desafios para a promoção da saúde. Rev Bras Enferm. [Internet] 2009;62(1) [acesso em 08 jan 2012] Disponível: http:// dx.doi.org/10.1590/S0034-71672009000100013

10. Oliveira DLLC. A enfermagem e suas apostas no autocuidado: investimentos emancipatórios ou práticas de sujeição? Rev. bras. enferm. [Internet] 2011;64(1) [acesso em 15 jan 2012] Disponível: http://dx.doi. org/10.1590/S0034-71672011000100027

11. Almeida Filho N. Reconhecer Flexner: inquérito sobre produção de mitos na educação médica no Brasil contemporâneo. Cad. Saúde Pública. [Internet] 2010;26(12) [acesso em 15 jan 2012] Disponível: http:// dx.doi.org/10.1590/S0102-311X2010001200003

12. Rigon AG, Neves ET. Educação em saúde e a atuação de enfermagem no contexto de unidades de internação hospitalar: o que tem sido ou há para ser dito? Texto Contexto Enferm. [Internet] 2011;20(4) [acesso em 15 jan 2012]. Disponível: http://dx.doi.org/10.1590/S010407072011000400022

13. Kruse MHL. Os poderes dos corpos frios: das coisas que ensinam às enfermeiras [tese]. Porto Alegre (RS): Universidade Federal do rio Grande do Sul; 2003.

14. Chagas RN, Ramos IC, Silva LF, Macedo ARM, Melo AVF. Cuidado crítico e criativo: contribuições da educação conscientizadora de Paulo Freire para a enfermagem. Ciência y Enfermeria. [Internet] 2009;(2) [acesso em 05 fev 2012]. Disponível: http://dx.doi. org/10.4067/S0717-95532009000200005

15. Freire P. Pedagogia do oprimido. 47a ed. Rio de Janeiro: Paz e Terra; 2005.
16. Castilho NC, Ribeiro PC, Chirelli MQ. A implementação da sistematização da assistência de enfermagem no serviço de saúde hospitalar no Brasil. Texto Contexto Enferm. [Internet] 2009;18(2) [acesso em $20 \mathrm{fev}$ 2012]. Disponível: http://dx.doi.org/10.1590/S010407072009000200011

17. Maciel MED. Educação em saúde: conceitos e propósitos. Cogitare enferm. 2009;14(4):773-6.

18. Pinheiro ALU, Beuter M, Brondani CM, Roso CC, Flores RG. Humanização no cuidado hospitalar: percepção de familiares acompanhantes. REUFSM [Internet] 2011;1(2) [acesso em 07 mai 2012]. Disponível: http:/cascavel.ufsm.br/revistas/ojs-2.2.2/index.php/ reufsm/article/view/2525 